\title{
Forced Vibration Numerical Analysis of Rectangular Elastic Orthotropic Damped Inclined Mindlin Plate Using Finite Difference Algorithm
}

\author{
Michael C. Agarana1,2, Amenawon I. Ehigbochie ${ }^{3}$ \\ ${ }^{1}$ Department of Mathematics, Covenant University, Ota, Nigeria \\ ${ }^{2}$ Department of Mechanical Engineering Science, University of Johannesburg, Johannesburg, South Africa \\ ${ }^{3}$ Department of Computer Science and Management Information System, Covenant University, Ota, Nigeria \\ Email: michael.agarana@covenantuniversity.edu.ng
}

How to cite this paper: Agarana, M.C. and Ehigbochie, A.I. (2018) Forced Vibration Numerical Analysis of Rectangular Elastic Orthotropic Damped Inclined Mindlin Plate Using Finite Difference Algorithm. Applied Mathematics, 9, 618-632. https://doi.org/10.4236/am.2018.96043

Received: February 13, 2018

Accepted: June 16, 2018

Published: June 19, 2018

Copyright (c) 2018 by authors and Scientific Research Publishing Inc. This work is licensed under the Creative Commons Attribution International License (CC BY 4.0).

http://creativecommons.org/licenses/by/4.0/

\begin{abstract}
Plates vibrate when load moves on them. In this paper, the dynamic response of Mindlin plate analytical model was converted to its numerical form using finite difference algorithm. The numerical model was analysed to ascertain the critical parameters contributing to the deflection of Mindlin plate under a moving load. The examination was more reasonable as in the likelihood of the plate laying on a Pasternak foundation was put into thought. Likewise the impact of damping was not dismissed. The plate considered in this paper was an inclined Mindlin plate, where the impacts of shear deformation and rotatory inertia were considered. The numerical equations were solved with the help of a developed computer program and Matlab. The results were consistent with what we have in the literature. The effects of the Pasternak foundation, damping, angle of inclination, and the moving load to the dynamic response of the elastic plate were exceptionally self-evident.
\end{abstract}

\section{Keywords}

Load, Plate, Algorithm, Mindlin, Damping, Analysis

\section{Introduction}

The importance of moving load problem manifested in numerous applications in the area of Mathematics and Engineering. Structural elements are usually designed to support moving loads. Various authors have analysed the dynamic 
response of a Mindlin Elastic plate under the influence of moving load, without considering the influence of inclination with the rotatory inertia and shear deformation on the plate. This is necessary because of the practical application in modern Engineering [1]-[7]. A load put on a slanted plane will frequently slide down the surface if nothing is keeping it down, particularly when the sliding surface (plate) is frictionless or practically frictionless. Likewise, the rate at which the object slides down the plate depends on the edge of slant of the surface; the more noteworthy the edge of slant of the surface, the quicker the rate at which the load will slide down it [8]. The edge of slant is the edge plate makes with the level. There are always, at least, two forces namely: the force of gravity and the normal force, acting upon the railway vehicle positioned on an inclined bridge [9]. The force of gravity acts in a downward direction, while the normal force acts in a direction perpendicular to the surface [10]. An inclined plane problem is in every way like any other net force problem with the sole exception that the surface has been tilted. An inclined plane therefore can be transformed into the form with which we are more comfortable [11]. After this transformation, one can ignore the force of gravity since it has been replaced by its two components and solve for the net force and the acceleration for a load mowing down the inclined plate [11]. Mindlin plate is the sort that put into thought the impact of shear deformation and rotatory inertia. Damping was considered in this work. The plate considered is of wood material, an example of orthotropic material. It has material properties that contrast along three commonly orthogonal twofold axes of rotational symmetry. It is a subset of anisotropic materials, since its properties change when measured from different directions [12] [13]. It has material properties that differ along three mutually-orthogonal twofold axes of rotational symmetry. It is a subset of anisotropic materials, because its properties change when measured from different directions [13]. A versatile rectangular inclined orthotropic Mindlin plate, supported by Pasternak foundation and traversed by a descending moving load is considered [14]. Loads are known to accelerate down slanted planes in view of a lopsided force [14]. Because of the orthotropic plate, the impact of contact, expressed by friction, is put into thought. The perpendicular component of the force of gravity is directed opposite the normal force and as such balances the normal force. The parallel component of the force of gravity is not balanced by any other force. This object will subsequently accelerate down the inclined plane due to the presence of an unbalanced force. It is the parallel component of the force of gravity that causes this acceleration. The parallel component of the force of gravity is the net force. In the presence of friction, determining the net force, which is the vector sum of all the forces, is as follows: The perpendicular component of force balances the normal force since objects do not accelerate perpendicular to the incline. The perpendicular component and the normal force add to $0 \mathrm{~N}$. The parallel component and the friction force add together to a magnitude greater than $0 \mathrm{~N}$, since we are considering a moving load. In this 
paper, finite difference algorithm was utilized to model the differential equations governing the dynamic response of an elastic rectangular damped inclined orthotropic Mindlin plate supported by a Pasternak foundation to a downward moving load. The algorithm converted the equations to their algebraic forms.

\section{Problem Formulation}

\subsection{Assumptions}

1) The inclined plate is of constant cross-section,

2) The moving load moves with a constant speed,

3) The moving railway vehicle is guided in such a way that it keeps contact with the inclined plate throughout the motion,

4) The inclined plate is continuously supported by a Pasternak foundation,

5) The moving load is moving downwards,

6) The rectangular Mindlin plate is elastic,

7) There is no damping in the system,

8) Uniform gravitational field,

9) Constant mass of the load moving down the inclined plane,

10) Constant angle of inclination.

\subsection{Governing Equations}

The simplified form of the set of dynamic equilibrium equations governing the behaviour of damped simply supported orthotropic inclined Mindlin plate traversed by a partially distributed downward moving load is given as [15] [16] [17]:

$$
\begin{aligned}
& \frac{M_{L} B}{\gamma A}\left[g \sin \theta+\frac{\partial^{2} W}{\partial T^{2}}+2 U \frac{\partial^{2} W}{\partial x \partial T}+U^{2} \frac{\partial^{2} W}{\partial x^{2}}\right]-F_{S} \\
&= k^{2} G h\left[-\frac{\partial^{2} W}{\partial x^{2}}+\frac{\partial \psi_{x}}{\partial x}-\frac{\partial^{2} W}{\partial y^{2}}+\frac{\partial^{2} \psi_{y}}{\partial y}\right]-K W-M_{f} \frac{\partial^{2} W}{\partial T^{2}} \\
&-G_{1} \frac{\partial^{2} W}{\partial x^{2}}-G_{1} \frac{\partial^{2} W}{\partial y^{2}}+\rho h \frac{\partial^{2} W}{\partial T^{2}} \\
& \frac{B_{\rho L} h_{1}^{3}}{12}\left[\frac{\partial^{2} \psi_{x}}{\partial T^{2}}+2 U \frac{\partial^{2} \psi_{x}}{\partial x \partial T}+U^{2} \frac{\partial^{2} \psi_{x}}{\partial x^{2}}\right]+\frac{\rho h^{3}}{12} \frac{\partial^{2} \psi_{x}}{\partial T^{2}} \\
&=D\left[\frac{\partial^{2} \psi_{y}}{\partial x^{2}}+v \frac{\partial^{2} \psi_{y}}{\partial x \partial y}\right]+\frac{1-v}{2} D\left[\frac{\partial^{2} \psi_{x}}{\partial y^{2}}+\frac{\partial^{2} \psi_{y}}{\partial x \partial y}\right]-k^{2} G h\left(\psi_{x}-\frac{\partial W}{\partial x}\right) \\
& \frac{B_{\rho L} h_{1}^{3}}{12}\left[\frac{\partial^{2} \psi_{y}}{\partial T^{2}}+2 U \frac{\partial^{2} \psi_{y}}{\partial y \partial T}+U^{2} \frac{\partial^{2} \psi_{y}}{\partial_{y}^{2}}\right]+\frac{\rho h^{3}}{12} \frac{\partial^{2} \psi_{y}}{\partial T^{2}} \\
&=D\left[\frac{\partial^{2} \psi_{y}}{\partial y^{2}}+v \frac{\partial^{2} \psi_{x}}{\partial y \partial x}\right]+\frac{1-v}{2} D\left[\frac{\partial^{2} \psi_{x}}{\partial x \partial y}+\frac{\partial^{2} \psi_{y}}{\partial x^{2}}\right]-k^{2} G h\left(\psi_{y}-\frac{\partial W}{\partial y}\right)
\end{aligned}
$$

The definitions for moments along $\mathrm{x}$ and $\mathrm{y}$ axes, twisting moment and shear deformation along $\mathrm{x}$ and $\mathrm{y}$ axes are given as follows respectively [17] [18] [19]

$$
M_{x}=-D\left(\frac{\partial \psi_{x}}{\partial x}+v \frac{\partial \psi_{y}}{\partial y}\right)
$$




$$
\begin{gathered}
M_{y}=-D\left(\frac{\partial \psi_{x}}{\partial y}+v \frac{\partial \psi_{x}}{\partial x}\right) \\
M_{x y}=-\frac{D(1-v)}{2}\left(\frac{\partial \psi_{x}}{\partial y}+\frac{\partial \psi_{y}}{\partial x}\right) \\
Q_{x}=-k^{2} G h\left(\psi_{x}-\frac{\partial W}{\partial x}\right) \\
Q_{y}=-k^{2} G h\left(\psi_{y}-\frac{\partial W}{\partial y}\right)
\end{gathered}
$$

where,

$\psi_{x}(x, y, T)$ and $\psi_{y}(x, y, T)$ are local rotation in the $x$ and $y$ directions respectively.

$W(x, y, T)$ is the traversed displacement of the plate at time $T$.

$g(\sin \theta)=$ acceleration due to gravity of the load down the inclined plane.

$\theta=$ angle of inclination of the plate to the horizontal.

$\gamma=$ damping coefficient.

$g(\cos \theta)=$ acceleration due to gravity of the load equal and opposite the normal force to the plane.

$F_{S}$ is the force of sliding friction.

$g$ is acceleration due to gravity.

$K, G_{1}$ = foundation stiffness.

$B=B_{x} B_{y}$ such that

$$
B_{X}=\left\{\begin{array}{lr}
1-H\left(x-\xi-\frac{\varepsilon}{2}\right), & 0 \leq T \leq \frac{\varepsilon}{U} \\
H\left(x-\xi+\frac{\varepsilon}{2}\right)-H\left(x-\xi-\frac{\varepsilon}{2}\right), & \frac{\varepsilon}{U} \leq T \leq \frac{L_{x}}{U} \\
H\left(x-\xi-\frac{\varepsilon}{2}\right), & \frac{L_{x}}{U} \leq T<\frac{L_{x}+\varepsilon}{U} \\
0, & \frac{L_{x}+\varepsilon}{U} \leq T \\
B_{y}=\left\{H\left(y-y_{1}+\frac{\mu}{2}\right)-H\left(y-y_{1}-\frac{\mu}{2}\right)\right\}
\end{array}\right.
$$

$H(x)$ is the Heaviside function defined as:

$$
H(x)= \begin{cases}1, & x>0 \\ 0.5, & x=0 \\ 0, & x<0\end{cases}
$$

$U$ is the velocity of a load of rectangular dimension $\varepsilon$ by $\mu$ with one of its line of symmetry moving along $Y=Y_{1}$.

$A=\mu \varepsilon$, the area of the load in contact with the plate.

The plate is $L_{x}$ by $L_{y}$ in dimensions and

$$
\xi=U T+\frac{\varepsilon}{2}
$$


$h$ and $h_{1}$ are thickness of the plate and load respectively.

$\rho$ and $\rho_{L}$ are the densities of the plate and load respectively.

$G$ is the modulus of the plate.

$D$ is the flexural rigidity of the plate defined by

$$
D=\frac{1}{2} E h^{2}\left[\left(1-v^{3}\right)\right]=G h^{3} / 6(1-v)
$$

$k^{2}$ is the shear correction factor.

$v$ is the poisson's ratio of the plate.

$g$ is the acceleration due to gravity.

$E$ is Young modulus of Elasticity.

$M_{L}$ is mass of the load.

\subsection{Boundary and Initial Conditions}

For a complete formulation of the problem, a simply supported rectangular Mindlin plate is considered as an illustrative example. If the edge $y=0$ of the simply supported, it then follows that the deflection $\mathrm{W}$ along this edge must be zero. At the same time this edge can rotate freely with respect to the $\mathrm{x}$-axis, i.e., there are no bending $\left(M_{x}\right)$ along this edge. Therefore the boundary conditions can be stated as follows: [19] [20] [21]

$$
\left.\begin{array}{l}
W(x, y, T)=M_{x}(x, y, T)=0, \text { for } x=0 \text { and } x=a \\
W(x, y, T)=M_{y}(x, y, T)=0, \text { for } y=0 \text { and } y=b
\end{array}\right\}
$$

The corresponding initial conditions are

$$
W(x, y, 0)=0=\frac{\partial W}{\partial T}(x, y, 0)
$$

\subsection{First Order PDE Version of the Governing Equations}

The first order partial differential equations versions of the system of Equations (1)-(8) are as follows:

$$
\begin{aligned}
& Q_{X}-\frac{\partial M_{X Y}}{\partial Y}-\frac{\partial M_{X}}{\partial X} \\
= & \frac{\rho h^{3}}{12} \frac{\partial \psi_{X, T}}{\partial T}+\frac{\rho_{L} h_{1}^{3}}{12}\left[\frac{\partial \psi_{Y, T}}{\partial T}+\frac{1}{D\left(v^{2}-1\right)} U\left(\frac{\partial M_{X}}{\partial T}+U \frac{\partial M_{X}}{\partial X}\right)\right. \\
& \left.-\frac{1}{D\left(v^{2}-1\right)} U\left(\frac{\partial M_{Y}}{\partial T}+U \frac{\partial M_{Y}}{\partial X}\right)\right] B \\
Q_{Y}-\frac{\partial M_{X Y}}{\partial X}-\frac{\partial M_{Y}}{\partial Y} & \frac{\rho h^{3}}{12} \frac{\partial \psi_{Y, T}}{\partial T}+\frac{\rho_{L} h_{1}^{3}}{12}\left[\frac{\partial \psi_{Y, T}}{\partial T}+U \frac{\partial \psi_{Y, T}}{\partial Y}+\frac{1}{D\left(v^{2}-1\right)} U\left(\frac{\partial M_{Y}}{\partial T}+U \frac{\partial M_{Y}}{\partial Y}\right)\right. \\
- & \left.\frac{U}{D\left(v^{2}-1\right)}\left(\frac{\partial M_{X}}{\partial T}+U \frac{\partial M_{X}}{\partial Y}\right)\right] B
\end{aligned}
$$




$$
\begin{aligned}
& \frac{\partial Q_{X}}{\partial X}+\frac{\partial Q_{Y}}{\partial Y}+K W+M \frac{\partial D_{T}}{\partial T}+G_{1} \frac{\partial D_{X}}{\partial X}+G_{1} \frac{\partial D_{Y}}{\partial Y} \\
& +\frac{M_{L}}{\gamma A}\left[g \sin \theta+\frac{\partial D_{T}}{\partial T}+U \frac{\partial D_{T}}{\partial T}+U\left\{\psi_{X, T}+\frac{U}{D\left(v^{2}-1\right)} M_{X}-\frac{U v}{D\left(v^{2}-1\right)} M_{Y}\right\}\right. \\
& \left.-\frac{U}{\alpha G h}\left\{\frac{\partial Q_{X}}{\partial T}+U \frac{\partial Q_{X}}{\partial X}\right\}\right] B=0
\end{aligned}
$$

where $M=M_{f}-\rho h$

$$
\begin{gathered}
\frac{\partial M_{X}}{\partial T}=-D \frac{\partial \psi_{X, T}}{\partial X}-D v \frac{\partial \psi_{Y, T}}{\partial Y} \\
\frac{\partial M_{Y}}{\partial T}=-D \frac{\partial \psi_{Y, T}}{\partial Y}-D v \frac{\partial \psi_{X, T}}{\partial X} \\
\frac{\partial M_{X Y}}{\partial T}=-D \frac{1-v}{2}\left[\frac{\partial \psi_{X, T}}{\partial Y}-\frac{\partial \psi_{Y, T}}{\partial X}\right] \\
\frac{\partial Q_{X}}{\partial T}=\alpha h G\left(\psi_{X, T}-\frac{\partial D_{T}}{\partial X}\right) \\
\frac{\partial Q_{Y}}{\partial T}=\alpha h G\left(\psi_{Y, T}-\frac{\partial D_{T}}{\partial Y}\right)
\end{gathered}
$$

where $W$ is the deflection

$$
\begin{aligned}
& \psi_{X, T}=\frac{\partial \psi_{X}}{\partial T} \\
& \psi_{Y, T}=\frac{\partial \psi_{Y}}{\partial T}
\end{aligned}
$$

and

$$
\begin{aligned}
D_{T} & =\frac{\partial W}{\partial T} \\
D_{X} & =\frac{\partial W}{\partial X} \\
D_{Y} & =\frac{\partial W}{\partial Y}
\end{aligned}
$$

\section{Finite Difference Algorithm for the Model}

Equations (16)-(26) were solved using a numerical method based on the finite difference algorithm. These equations were transformed into their equivalent algebraic forms. The finite difference definition of first order partial derivative of a function $E(x, y, t)$ say, with respect to $x, y$ and $t$ respectively are as follows [21] [22]:

$$
\begin{aligned}
& \frac{\partial E}{\partial t}=\frac{1}{4 r^{*}}\left[E_{i+1, j+1}^{k+1}+E_{i+1, j}^{k+1}+E_{i, j+1}^{k+1}+E_{i, j}^{k+1}-E_{i+1, j+1}^{k}-E_{i+1, j}^{k}-E_{i, j+1}^{k}-E_{i, j}^{k}\right] \\
& \frac{\partial E}{\partial x}=\frac{1}{4 h^{*}}\left[E_{i+1, j+1}^{k+1}+E_{i+1, j}^{k+1}-E_{i, j}^{k+1}-E_{i, j+1}^{k+1}+E_{i+1, j+1}^{k}+E_{i+1, j}^{k}-E_{i, j+1}^{k}-E_{i, j}^{k}\right]
\end{aligned}
$$




$$
\frac{\partial E}{\partial y}=\frac{1}{4 k^{*}}\left[E_{i+1, j+1}^{k+1}+E_{i, j+1}^{k+1}-E_{i+1, j}^{k+1}-E_{i, j}^{k+1}+E_{i+1, j+1}^{k}+E_{i, j+1}^{k}-E_{i+1, j}^{k}-E_{i, j}^{k}\right]
$$

where $E$ is the function value of the centre of a grid, which is well approximated by the average of its values at the grid nodes [22].

$$
\begin{aligned}
& E\left(x+\frac{h^{*}}{2}, y+\frac{k^{*}}{2}, t+\frac{r^{*}}{2}\right) \\
& =\frac{1}{8}\left[E_{i+1, j+1}^{k+1}+E_{i+1, j}^{k+1}+E_{i, j+1}^{k+1}+E_{i, j}^{k+1}+E_{i+1, j+1}^{k}+E_{i+1, j}^{k}+E_{i, j+1}^{k}+E_{i, j}^{k}\right]
\end{aligned}
$$

Using the above finite difference definition on Equations (16)-(26) gives:

$$
\begin{aligned}
& \frac{1}{8}\left[Q_{x i+1, j+1}^{k+1}+Q_{x i+1, j}^{k+1}+Q_{x i, j+1}^{k+1}+Q_{x i, j}^{k+1}+Q_{x i+1, j+1}^{k}+Q_{x i+1, j}^{k}+Q_{x i, j+1}^{k}+Q_{x i, j}^{k}\right] \\
& -\frac{1}{4 k^{*}}\left[M_{X Y i+1, j+1}^{k+1}+M_{X Y i, j+1}^{k+1}-M_{X Y i+1, j}^{k+1}-M_{X Y i, j}^{k+1}+M_{X Y i+1, j+1}^{k}\right. \\
& \left.+M_{X Y i, j+1}^{k}-M_{X Y i+1, j}^{k}-M_{X Y i, j}^{k}\right]-\frac{1}{4 h^{*}}\left[M_{X i+1, j+1}^{k+1}+M_{X i+1, j}^{k+1}\right. \\
& \left.-M_{X i, j}^{k+1}-M_{X i, j+1}^{k+1}+M_{X i+1, j+1}^{k}+M_{X i+1, j}^{k}-M_{X i, j+1}^{k}-M_{X i, j}^{k}\right] \\
& =\frac{\rho h^{3}}{12} \frac{1}{4 r^{*}}\left[\psi_{X, T i+1, j+1}^{k+1}+\psi_{X, T i+1, j}^{k+1}+\psi_{X, T i, j+1}^{k+1}+\psi_{X, T i, j}^{k+1}-\psi_{X, T i+1, j+1}^{k}-\psi_{X, T i+1, j}^{k}\right. \\
& \left.-\underset{X, T i, j+1}{k}-\psi_{X, T i, j}^{k}\right]+\frac{\rho_{L} h_{1}^{3} B}{12} \frac{1}{4 r^{*}}\left[\psi_{Y, T i+1, j+1}^{k+1}+\psi_{Y, T i+1, j}^{k+1}+\psi_{Y, T i, j+1}^{k+1}+\psi_{Y, T i, j}^{k+1}\right. \\
& \left.-\psi_{Y, T i+1, j+1}^{k}-\psi_{Y, T i+1, j}^{k}-\psi_{Y, T i, j+1}^{k}-\psi_{Y, T i, j}^{k}\right]+\frac{\rho h^{3}}{12} \frac{U B}{D\left(v^{2}-1\right)} \frac{1}{4 r^{*}}\left[M_{X i+1, j+1}^{k+1}\right. \\
& \left.+M_{X i+1, j}^{k+1}+M_{X i, j+1}^{k+1}+M_{X i, j}^{k+1}-M_{X i+1, j+1}^{k}-M_{X i+1, j}^{k}-M_{X i, j+1}^{k}-M_{X i, j}^{k}\right] \\
& +\frac{\rho h^{3}}{12} \frac{U^{2} B}{D\left(v^{2}-1\right)} \frac{1}{4 h^{*}}\left[M_{X i+1, j+1}^{k+1}+M_{X i+1, j}^{k+1}-M_{X i, j}^{k+1}-M_{X i, j+1}^{k+1}+M_{X i+1, j+1}^{k}\right. \\
& \left.+M_{X i+1, j}^{k}-M_{X i, j+1}^{k}-M_{X i, j}^{k}\right]-\frac{\rho h^{3}}{12} \frac{U B}{D\left(v^{2}-1\right)} \frac{1}{4 r^{*}}\left[M_{Y i+1, j+1}^{k+1}+M_{Y i+1, j}^{k+1}\right. \\
& \left.+M_{Y i, j+1}^{k+1}+M_{Y i, j}^{k+1}-M_{Y i+1, j+1}^{k}-M_{Y i+1, j}^{k}-M_{Y i, j+1}^{k}-M_{Y i, j}^{k}\right] \\
& -\frac{\rho h^{3}}{12} \frac{U^{2} B}{D\left(v^{2}-1\right)}\left\{\frac { 1 } { 4 h ^ { * } } \left[M_{Y i+1, j+1}^{k+1}+M_{Y i+1, j}^{k+1}-M_{Y i, j}^{k+1}\right.\right. \\
& \left.-M_{Y i, j+1}^{k+1}+M_{Y i+1, j+1}^{k}+M_{Y i+1, j}^{k}-M_{Y i, j+1}^{k}-M_{Y i, j}^{k}\right] \\
& \frac{1}{8}\left[Q_{Y i+1, j+1}^{k+1}+Q_{Y i+1, j}^{k+1}+Q_{Y i, j+1}^{k+1}+Q_{Y i, j}^{k+1}+Q_{Y i+1, j+1}^{k}+Q_{Y i+1, j}^{k}+Q_{Y i, j+1}^{k}+Q_{Y i, j}^{k}\right] \\
& -\frac{1}{4 h^{*}}\left[M_{X Y i+1, j+1}^{k+1}+M_{X Y i, j+1}^{k+1}-M_{X Y i+1, j}^{k+1}-M_{X Y i, j}^{k+1}+M_{X Y i+1, j+1}^{k}\right. \\
& \left.+M_{X Y i, j+1}^{k}-M_{X Y i+1, j}^{k}-M_{X Y i, j}^{k}\right]-\frac{1}{4 k^{*}}\left[M_{X i+1, j+1}^{k+1}+M_{X i+1, j}^{k+1}\right. \\
& \left.-M_{X i, j}^{k+1}-M_{X i, j+1}^{k+1}+M_{X i+1, j+1}^{k}+M_{X i+1, j}^{k}-M_{X i, j+1}^{k}-M_{X i, j}^{k}\right]
\end{aligned}
$$




$$
\begin{aligned}
& =\frac{\rho h^{3}}{12} \frac{1}{4 r^{*}}\left[\begin{array}{c}
k+1 \\
Y, T i+1, j+1
\end{array}+\psi_{Y, T i+1, j}^{k+1}+\psi_{Y, T i, j+1}^{k+1}+\psi_{Y, T i, j}^{k+1}-\psi_{Y, T i+1, j+1}^{k}-\psi_{Y, T i+1, j}^{k}\right. \\
& \left.-\psi_{Y, T i, j+1}^{k}-\psi_{Y, T i, j}^{k}\right]+\frac{\rho_{L} h_{1}^{3} B}{12} \frac{1}{4 r^{*}}\left[\begin{array}{c}
k+1 \\
\psi_{Y, T i+1, j+1}^{k}
\end{array}+\psi_{Y, T i+1, j}^{k+1}+\psi_{Y, T i, j+1}^{k+1}+\psi_{Y, T i, j}^{k+1}\right. \\
& \left.-\psi_{Y, T i+1, j+1}^{k}-\psi_{Y, T i+1, j}^{k}-\psi_{Y, T i, j+1}^{k}-\psi_{Y, T i, j}^{k}\right]+\frac{\rho h^{3} U B}{12} \frac{1}{4 k^{*}}\left[\psi_{Y, T i+1, j+1}^{k+1}+\psi_{Y, T i+1, j}^{k+1}\right. \\
& \left.-\psi_{Y, T i, j}^{k+1}-\psi_{Y, T i, j+1}^{k+1}+\psi_{Y, T i+1, j+1}^{k}+\psi_{Y, T i+1, j}^{k}-\psi_{Y, T i, j+1}^{k}-\psi_{Y, T i, j}^{k}\right] \\
& +\frac{\rho h^{3}}{12} \frac{U B}{D\left(v^{2}-1\right)} \frac{1}{4 r^{*}}\left[M_{X i+1, j+1}^{k+1}+M_{X i+1, j}^{k+1}+M_{X i, j+1}^{k+1}+M_{X i, j}^{k+1}-M_{X i+1, j+1}^{k}\right. \\
& \left.-M_{X i+1, j}^{k}-M_{X i, j+1}^{k}-M_{X i, j}^{k}\right]+\frac{\rho h^{3}}{12} \frac{U^{2} B}{D\left(v^{2}-1\right)} \frac{1}{4 k^{*}}\left[M_{X i+1, j+1}^{k+1}+M_{X i+1, j}^{k+1}\right. \\
& \left.-M_{X i, j}^{k+1}-M_{X i, j+1}^{k+1}+M_{X i+1, j+1}^{k}+M_{X i+1, j}^{k}-M_{X i, j+1}^{k}-M_{X i, j}^{k}\right] \\
& -\frac{\rho h^{3}}{12} \frac{U B}{D\left(v^{2}-1\right)} \frac{1}{4 r^{*}}\left[M_{X i+1, j+1}^{k+1}+M_{X i+1, j}^{k+1}+M_{X i, j+1}^{k+1}+M_{X i, j}^{k+1}-M_{X i+1, j+1}^{k}\right. \\
& \left.-M_{X i+1, j}^{k}-M_{X i, j+1}^{k}-M_{X i, j}^{k}\right]-\frac{\rho h^{3}}{12} \frac{U^{2} B}{D\left(v^{2}-1\right)} \frac{1}{4 h^{*}}\left[M_{X i+1, j+1}^{k+1}+M_{X i+1, j}^{k+1}\right. \\
& \left.-M_{X i, j}^{k+1}-M_{X i, j+1}^{k+1}+M_{X i+1, j+1}^{k}+M_{X i+1, j}^{k}-M_{X i, j+1}^{k}-M_{X i, j}^{k}\right] \\
& \frac{1}{4 h^{*}}\left[Q_{x i+1, j+1}^{k+1}+Q_{x i+1, j}^{k+1}-Q_{x i, j}^{k+1}-Q_{x i, j+1}^{k+1}+Q_{x i+1, j+1}^{k}+Q_{x i+1, j}^{k}-Q_{x i, j+1}^{k}-Q_{x i, j}^{k}\right] \\
& +\frac{1}{4 k^{*}}\left[Q_{x i+1, j+1}^{k+1}+Q_{x i, j+1}^{k+1}-Q_{x i+1, j}^{k+1}-Q_{x i, j}^{k+1}+Q_{x i+1, j+1}^{k}+Q_{x i, j+1}^{k}-Q_{x i+1, j}^{k}-Q_{x i, j}^{k}\right] \\
& +\frac{1}{8} K\left[W_{x i+1, j+1}^{k+1}+W_{x i, j+1}^{k+1}+W_{x i+1, j}^{k+1}+W_{x i, j}^{k+1}+W_{x i+1, j+1}^{k}+W_{x i, j+1}^{k}+W_{x i+1, j}^{k}+W_{x i, j}^{k}\right] \\
& +M \frac{1}{4 r^{*}}\left[D_{T i+1, j+1}^{k+1}+D_{T i+1, j}^{k+1}+D_{T i, j}^{k+1}+D_{T i, j+1}^{k+1}-D_{T i+1, j+1}^{k}-D_{T i+1, j}^{k}-D_{T i, j+1}^{k}-D_{T i, j}^{k}\right] \\
& +G_{1} \frac{1}{4 h^{*}}\left[D_{x i+1, j+1}^{k+1}+D_{x i+1, j}^{k+1}-D_{x i, j}^{k+1}-D_{x i, j+1}^{k+1}+D_{x i+1, j+1}^{k}+D_{x i+1, j}^{k}-D_{x i, j+1}^{k}-D_{x i, j}^{k}\right] \\
& +G_{1} \frac{1}{4 k^{*}}\left[D_{Y i+1, j+1}^{k+1}+D_{Y i, j+1}^{k+1}-D_{Y i+1, j}^{k+1}-D_{Y i, j}^{k+1}+D_{Y i+1, j+1}^{k}+D_{Y i, j+1}^{k}-D_{Y i+1, j}^{k}-D_{Y i, j}^{k}\right] \\
& +\frac{M_{L}}{\gamma A}\left[g \sin \theta+\frac{1}{4 r^{*}}\left[D_{T i+1, j+1}^{k+1}+D_{T i+1, j}^{k+1}+D_{T i, j}^{k+1}+D_{T i, j+1}^{k+1}-D_{T i+1, j+1}^{k}-D_{T i+1, j}^{k}\right.\right. \\
& \left.\left.-D_{T i, j+1}^{k}-D_{T i, j}^{k}\right]\right] B+U \frac{M_{L}}{\gamma A} \frac{1}{4 r^{*}}\left[D_{T i+1, j+1}^{k+1}+D_{T i+1, j}^{k+1}+D_{T i, j}^{k+1}+D_{T i, j+1}^{k+1}-D_{T i+1, j+1}^{k}\right. \\
& \left.-D_{T i+1, j}^{k}-D_{T i, j+1}^{k}-D_{T i, j}^{k}\right] B+U \frac{M_{L}}{\gamma A} \frac{1}{8}\left[\psi_{X, T i+1, j+1}^{k+1}+\psi_{X, T i, j+1}^{k+1}+\psi_{X, T i+1, j}^{k+1}+\psi_{X, T i, j}^{k+1}\right. \\
& \left.+\psi_{X, T i+1, j+1}^{k}+\psi_{X, T i, j+1}^{k}+\psi_{X, T i+1, j}^{k}+\psi_{X, T i, j}^{k}\right] B+\frac{M_{L}}{\gamma A} \frac{U^{2}}{D\left(v^{2}-1\right)} \frac{1}{8}\left[M_{x i+1, j+1}^{k+1}\right. \\
& \left.+M_{x i, j+1}^{k+1}+M_{x i+1, j}^{k+1}+M_{x i, j}^{k+1}+M_{x i+1, j+1}^{k}+M_{x i, j+1}^{k}+M_{x i+1, j}^{k}+M_{x i, j}^{k}\right] B \\
& -\frac{M_{L}}{\gamma A} \frac{U^{2} v}{D\left(v^{2}-1\right)} \frac{1}{8}\left[M_{Y i+1, j+1}^{k+1}+M_{Y i, j+1}^{k+1}+M_{Y i+1, j}^{k+1}+M_{Y i, j}^{k+1}+M_{Y i+1, j+1}^{k}\right. \\
& \left.+M_{Y i, j+1}^{k}+M_{Y i+1, j}^{k}+M_{Y i, j}^{k}\right] B-\frac{M_{L}}{\gamma A} \frac{U}{\alpha G h} \frac{1}{4 r^{*}}\left[Q_{x i+1, j+1}^{k+1}+Q_{x i+1, j}^{k+1}+Q_{x i, j+1}^{k+1}\right. \\
& \left.+Q_{x i, j}^{k+1}-Q_{x i+1, j+1}^{k}-Q_{x i+1, j}^{k}-Q_{x i, j+1}^{k}-Q_{x i, j}^{k}\right] B-\frac{M_{L}}{\gamma A} \frac{U^{2}}{\alpha G h} \frac{1}{4 h^{*}}\left[Q_{Y i+1, j+1}^{k+1}+Q_{Y i+1, j}^{k+1}\right. \\
& \left.-Q_{Y i, j+1}^{k+1}-Q_{Y i, j}^{k+1}+Q_{Y i+1, j+1}^{k}+Q_{Y i+1, j}^{k}-Q_{Y i, j+1}^{k}-Q_{Y i, j}^{k}\right] B=0
\end{aligned}
$$




$$
\begin{aligned}
& \frac{1}{4 r^{*}}\left[M_{x i+1, j+1}^{k+1}+M_{x i+1, j}^{k+1}+M_{x i, j+1}^{k+1}+M_{x i, j}^{k+1}-M_{x i+1, j+1}^{k}-M_{x i+1, j}^{k}-M_{x i, j+1}^{k}-M_{x i, j}^{k}\right] \\
& =-D\left[\frac { 1 } { 4 h ^ { * } } \left[\psi_{X, T i+1, j+1}^{k+1}+\psi_{X, T i+1, j}^{k+1}-\psi_{X, T i, j}^{k+1}-\psi_{X, T i, j+1}^{k+1}+\psi_{X, T i+1, j+1}^{k}\right.\right. \\
& \left.\left.+\psi_{X, T i+1, j}^{k}-\psi_{X, T i, j+1}^{k}-\psi_{X, T i, j}^{k}\right]\right]-D v\left[\frac { 1 } { 4 k ^ { * } } \left[\begin{array}{c}
k+1 \\
\psi_{X, T i+1, j+1}^{k}
\end{array}+\psi_{X, T i, j+1}^{k+1}\right.\right. \\
& \left.\left.-\psi_{X, T i+1, j}^{k+1}-\psi_{X, T i, j}^{k+1}+\psi_{X, T i+1, j+1}^{k}+\psi_{X, T i, j+1}^{k}-\psi_{X, T i+1, j}^{k}-\psi_{X, T i, j}^{k}\right]\right] \\
& \frac{1}{4 r^{*}}\left[M_{Y i+1, j+1}^{k+1}+M_{Y i+1, j}^{k+1}+M_{Y i, j+1}^{k+1}+M_{Y i, j}^{k+1}-M_{Y i+1, j+1}^{k}-M_{Y i+1, j}^{k}-M_{Y i, j+1}^{k}-M_{Y i, j}^{k}\right]
\end{aligned}
$$

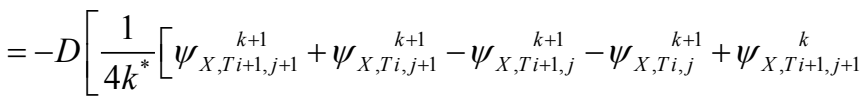

$$
\begin{aligned}
& \left.\left.+\psi_{X, T i, j+1}^{k}-\psi_{X, T i+1, j}^{k}-\psi_{X, T i, j}^{k}\right]\right]-D v\left[\frac { 1 } { 4 h ^ { * } } \left[\psi_{X, T i+1, j+1}^{k+1}+\psi_{X, T i, j+1}^{k+1}\right.\right. \\
& \left.\left.-\psi_{X, T i+1, j}^{k+1}-\psi_{X, T i, j}^{k+1}+\psi_{X, T i+1, j+1}^{k}+\psi_{X, T i, j+1}^{k}-\psi_{X, T i+1, j}^{k}-\psi_{X, T i, j}^{k}\right]\right] \\
& \frac{1}{4 r^{*}}\left[M_{X Y i+1, j+1}^{k+1}+M_{Y i+1, j}^{k+1}+M_{Y i, j+1}^{k+1}+M_{Y i, j}^{k+1}-M_{Y i+1, j+1}^{k}-M_{Y i+1, j}^{k}-M_{Y i, j+1}^{k}-M_{Y i, j}^{k}\right] \\
& =-D \frac{1-v}{2}\left[\frac { 1 } { 4 k ^ { * } } \left[\psi_{X, T i+1, j+1}^{k+1}+\psi_{X, T i, j+1}^{k+1}-\psi_{X, T i+1, j}^{k+1}-\psi_{X, T i, j}^{k+1}+\psi_{X, T i+1, j+1}^{k}\right.\right. \\
& \left.\left.+\psi_{X, T i, j+1}^{k}-\psi_{X, T i+1, j}^{k}-\psi_{X, T i, j}^{k}\right]\right]+D \frac{1-v}{2}\left[\frac { 1 } { 4 h ^ { * } } \left[\psi_{Y, T i+1, j+1}^{k+1}+\psi_{Y, T i, j+1}^{k+1}\right.\right. \\
& \left.\left.-\psi_{Y, T i+1, j}^{\stackrel{k+1}{k}}-\psi_{Y, T i, j}^{k+1}+\underset{Y, T i+1, j+1}{k}+\psi_{Y, T i, j+1}^{k}-\psi_{Y, T i+1, j}^{k}-\psi_{Y, T i, j}^{k}\right]\right] \\
& \frac{1}{4 r^{*}}\left[Q_{x i+1, j+1}^{k+1}+Q_{x i+1, j}^{k+1}+Q_{x i, j}^{k+1}+Q_{x i, j+1}^{k+1}-Q_{x i+1, j+1}^{k}-Q_{x i+1, j}^{k}-Q_{x i, j+1}^{k}-Q_{x i, j}^{k}\right] \\
& =\alpha h G\left[\frac { 1 } { 8 } \left[\psi_{X, T i+1, j+1}^{k+1}+\psi_{X, T i+1, j}^{k+1}+\psi_{X, T i, j}^{k+1}+\psi_{X, T i, j+1}^{k+1}+\psi_{X, T i+1, j+1}^{k}\right.\right. \\
& \left.\left.+\psi_{X, T i+1, j}^{k}+\psi_{X, T i, j+1}^{k}+\psi_{X, T i, j}^{k}\right]\right]-\alpha h G\left[\frac { 1 } { 4 h ^ { * } } \left[D_{Y i+1, j+1}^{k+1}+D_{Y i+1, j}^{k+1}\right.\right. \\
& \left.\left.-D_{Y i, j}^{k+1}-D_{Y i, j+1}^{k+1}+D_{Y i+1, j+1}^{k}+D_{Y i+1, j}^{k}-D_{Y i, j+1}^{k}-D_{Y i, j}^{k}\right]\right] \\
& \frac{1}{4 r^{*}}\left[Q_{Y i+1, j+1}^{k+1}+Q_{Y i+1, j}^{k+1}+Q_{Y i, j}^{k+1}+Q_{Y i, j+1}^{k+1}-Q_{Y i+1, j+1}^{k}-Q_{Y i+1, j}^{k}-Q_{Y i, j+1}^{k}-Q_{Y i, j}^{k}\right] \\
& =\alpha h G\left[\frac { 1 } { 8 } \left[\psi_{Y, T i+1, j+1}^{k+1}+\psi_{Y, T i+1, j}^{k+1}+\psi_{Y, T i, j}^{k+1}+\psi_{Y, T i, j+1}^{k+1}+\psi_{Y, T i+1, j+1}^{k}\right.\right. \\
& \left.\left.+\psi_{Y, T i+1, j}^{k}+\psi_{Y, T i, j+1}^{k}+\psi_{Y, T i, j}^{k}\right]\right]-\alpha h G\left[\frac { 1 } { 4 k ^ { * } } \left[D_{Y i+1, j+1}^{k+1}+D_{Y i, j+1}^{k+1}\right.\right. \\
& \left.\left.-D_{Y i+1, j}^{k+1}-D_{Y i, j}^{k+1}+D_{Y i+1, j+1}^{k}+D_{Y i, j+1}^{k}-D_{Y i+1, j}^{k}-D_{Y i, j}^{k}\right]\right] \\
& \frac{1}{8}\left[D_{T i+1, j+1}^{k+1}+D_{T i+1, j}^{k+1}+D_{T i, j}^{k+1}+D_{T i, j+1}^{k+1}-D_{T i+1, j+1}^{k}-D_{T i+1, j}^{k}-D_{T i, j+1}^{k}-D_{T i, j}^{k}\right] \\
& =\frac{1}{4 r^{*}}\left[W_{x i+1, j+1}^{k+1}+W_{x i, j+1}^{k+1}+W_{x i+1, j}^{k+1}+W_{x i, j}^{k+1}-W_{x i+1, j+1}^{k}-W_{x i, j+1}^{k}-W_{x i+1, j}^{k}-W_{x i, j}^{k}\right]
\end{aligned}
$$




$$
\begin{aligned}
& \frac{1}{8}\left[D_{Y i+1, j+1}^{k+1}+D_{Y i+1, j}^{k+1}+D_{Y i, j}^{k+1}+D_{Y i, j+1}^{k+1}+D_{Y i+1, j+1}^{k}+D_{Y i+1, j}^{k}+D_{Y i, j+1}^{k}+D_{Y i, j}^{k}\right] \\
& =\frac{1}{4 h^{*}}\left[W_{x i+1, j+1}^{k+1}+W_{x i+1, j}^{k+1}-W_{x i, j}^{k+1}-W_{x i, j+1}^{k+1}+W_{x i+1, j+1}^{k}+W_{x i+1, j}^{k}-W_{x i, j+1}^{k}-W_{x i, j}^{k}\right] \\
& \frac{1}{8}\left[D_{T i+1, j+1}^{k+1}+D_{T i+1, j}^{k+1}+D_{T i, j}^{k+1}+D_{T i, j+1}^{k+1}+D_{T i+1, j+1}^{k}+D_{T i+1, j}^{k}+D_{T i, j+1}^{k}+D_{T i, j}^{k}\right] \\
& =\frac{1}{4 k^{*}}\left[W_{x i+1, j+1}^{k+1}+W_{x i, j+1}^{k+1}-W_{x i+1, j}^{k+1}-W_{x i, j}^{k+1}+W_{x i+1, j+1}^{k}+W_{x i, j+1}^{k}-W_{x i+1, j}^{k}-W_{x i, j}^{k}\right]
\end{aligned}
$$

The set of algebraic equations to be solved may be written in matrix form as:

$$
\begin{aligned}
& R_{i, j+1} S_{i, j+1}^{\prime}+P_{i+1, j+1} S_{i+1, j+1}^{\prime}=-T_{i, j+1} S_{i, j+1}^{\prime}-Y_{i+1, j} S_{i+1, j}^{\prime}+Z_{k} \\
& i=1,2,3, \cdots, N-1, j=1,2,3, \cdots, M-1
\end{aligned}
$$

where $N$ and $M$ are the number of the modal points along $x$ - and $y$-axes respectively, $Z_{k}$ is a matrix representing the right half of Equations (16)-(26) defined by

$$
Z_{k}=A_{i, j} S_{i, j}^{0}+B_{i, j+1} S_{i, j+1}^{0}+C_{i+1, j} S_{i+1, j}^{0}+D_{i+1, j+1} S_{i+1, j+1}^{0}+E_{1}
$$

The terms of the above Equations ((42) and (43)) can be represented in matrix form as follows:

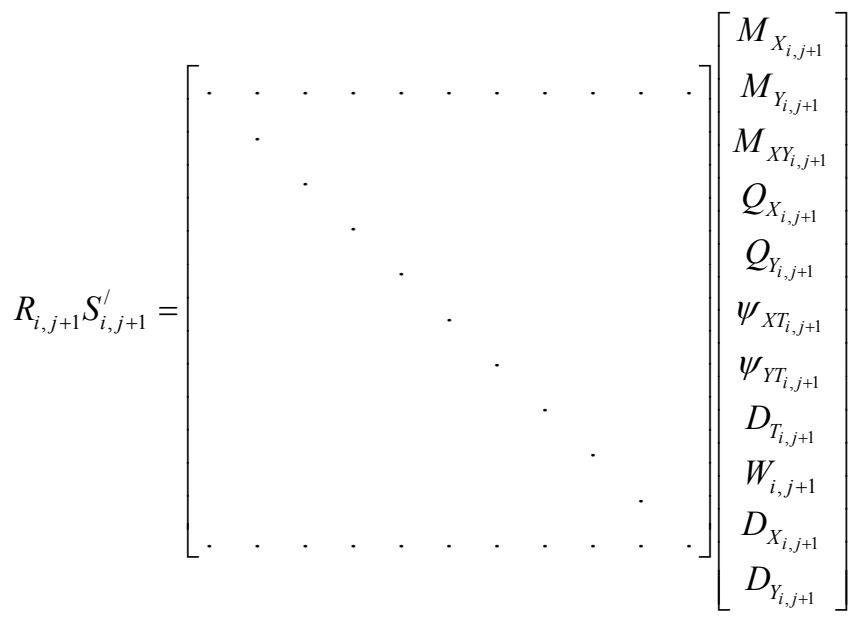

\section{Numerical Simulation}

For numerical work the coupled differential Equations (16)-(26) were solved using the central difference formula of finite difference method. The following values of the various parameters were used: $h=1, h_{1}=0.2, \rho=0.8, \rho_{L}=0.5, B=$ $0.5, U=5.5, D=0.63, v=0.2, M=10, M_{L}=0.05, K=100, G_{1}=10, \gamma=0.5, A=6$, $g=9.8, \theta=30, \alpha=0.01, G=200, r^{*}=1, h^{*}=1, k^{*}=1, r=2$. Equations (31) to (41) can now be written as follows:

$$
\begin{aligned}
& 0.125\left[Q_{x i+1, j+1}^{k+1}+Q_{x i+1, j}^{k+1}+Q_{x i, j+1}^{k+1}+Q_{x i, j}^{k+1}+Q_{x i+1, j+1}^{k}+Q_{x i+1, j}^{k}+Q_{x i, j+1}^{k}+Q_{x i, j}^{k}\right] \\
& -0.25\left[M_{X Y i+1, j+1}^{k+1}+M_{X Y i, j+1}^{k+1}-M_{X Y i+1, j}^{k+1}-M_{X Y i, j}^{k+1}+M_{X Y i+1, j+1}^{k}\right. \\
& \left.+M_{X Y i, j+1}^{k}-M_{X Y i+1, j}^{k}-M_{X Y i, j}^{k}\right]-0.25\left[M_{X i+1, j+1}^{k+1}+M_{X i+1, j}^{k+1}\right. \\
& \left.-M_{X i, j}^{k+1}-M_{X i, j+1}^{k+1}+M_{X i+1, j+1}^{k}+M_{X i+1, j}^{k}-M_{X i, j+1}^{k}-M_{X i, j}^{k}\right]
\end{aligned}
$$




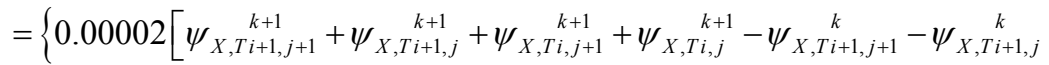

$$
\begin{aligned}
& \left.\left.-\psi_{X, T i, j+1}^{k}-\psi_{X, T i, j}^{k}\right]\right\}+\left\{0 . 0 0 0 7 5 \left[\begin{array}{c}
\psi_{Y, T i+1, j+1}^{k+1} \\
\stackrel{k+1}{k+1}+\psi_{Y, T i+1, j}+\psi_{Y, T i, j+1}^{k+1}+\psi_{Y, T i, j}^{k+1}
\end{array}\right.\right. \\
& \left.\left.-\psi_{Y, T i+1, j+1}^{k}-\psi_{Y, T i+1, j}^{k}-\psi_{Y, T i, j+1}^{k}-\psi_{Y, T i, j}^{k}\right]\right\}-\left\{0 . 0 0 1 2 1 \left[M_{X i+1, j+1}^{k+1}+M_{X i+1, j}^{k+1}\right.\right. \\
& \left.\left.+M_{X i, j+1}^{k+1}+M_{X i, j}^{k+1}-M_{X i+1, j+1}^{k}-M_{X i+1, j}^{k}-M_{X i, j+1}^{k}-M_{X i, j}^{k}\right]\right\} \\
& -\left\{0 . 0 0 0 8 \left[M_{X i+1, j+1}^{k+1}+M_{X i+1, j}^{k+1}-M_{X i, j}^{k+1}-M_{X i, j+1}^{k+1}+M_{X i+1, j+1}^{k}+M_{X i+1, j}^{k}\right.\right. \\
& \left.\left.-M_{X i, j+1}^{k}-M_{X i, j}^{k}\right]\right\}+\left\{0 . 0 0 1 2 1 \left[M_{Y i+1, j+1}^{k+1}+M_{Y i+1, j}^{k+1}+M_{Y i, j+1}^{k+1}+M_{Y i, j}^{k+1}\right.\right. \\
& \left.\left.-M_{Y i+1, j+1}^{k}-M_{Y i+1, j}^{k}-M_{Y i, j+1}^{k}-M_{Y i, j}^{k}\right]\right\}+\left\{0 . 0 0 6 6 7 \left[M_{Y i+1, j+1}^{k+1}+M_{Y i+1, j}^{k+1}\right.\right. \\
& \left.\left.-M_{Y i, j}^{k+1}-M_{Y i, j+1}^{k+1}+M_{Y i+1, j+1}^{k}+M_{Y i+1, j}^{k}-M_{Y i, j+1}^{k}-M_{Y i, j}^{k}\right]\right\} \\
& 0.125\left[Q_{Y i+1, j+1}^{k+1}+Q_{Y i+1, j}^{k+1}+Q_{Y i, j+1}^{k+1}+Q_{Y i, j}^{k+1}+Q_{Y i+1, j+1}^{k}+Q_{Y i+1, j}^{k}+Q_{Y i, j+1}^{k}+Q_{Y i, j}^{k}\right] \\
& -0.25\left[M_{X Y i+1, j+1}^{k+1}+M_{X Y i, j+1}^{k+1}-M_{X Y i+1, j}^{k+1}-M_{X Y i, j}^{k+1}+M_{X Y i+1, j+1}^{k}\right. \\
& \left.+M_{X Y i, j+1}^{k}-M_{X Y i+1, j}^{k}-M_{X Y i, j}^{k}\right]-0.25\left[M_{X i+1, j+1}^{k+1}+M_{X i+1, j}^{k+1}\right. \\
& \left.-M_{X i, j}^{k+1}-M_{X i, j+1}^{k+1}+M_{X i+1, j+1}^{k}+M_{X i+1, j}^{k}-M_{X i, j+1}^{k}-M_{X i, j}^{k}\right] \\
& =0.0667\left\{\frac { 1 } { 4 r } \left[\psi_{Y, T i+1, j+1}^{k+1}+\psi_{Y, T i+1, j}^{k+1}+\psi_{Y, T i, j+1}^{k+1}+\psi_{Y, T i, j}^{k+1}-\psi_{Y, T i+1, j+1}^{k}-\psi_{Y, T i+1, j}^{k}\right.\right. \\
& \left.\left.-\psi_{Y, T i, j+1}^{k}-\psi_{Y, T i, j}^{k}\right]\right\}+0.00002\left\{\frac { 1 } { 4 r } \left[\psi_{Y, T i+1, j+1}^{k+1}+\psi_{Y, T i+1, j}^{k+1}+\psi_{Y, T i, j+1}^{k+1}+\psi_{Y, T i, j}^{k+1}\right.\right. \\
& \left.\left.-\psi_{Y, T i+1, j+1}^{k}-\psi_{Y, T i+1, j}^{k}-\psi_{Y, T i, j+1}^{k}-\psi_{Y, T i, j}^{k}\right]\right\}+0.00046\left[\begin{array}{c}
k+1 \\
\psi_{Y, T i+1, j+1}^{k}+\psi_{Y, T i+1, j}^{k+1}-\psi_{Y, T i, j}^{k+1}
\end{array}\right. \\
& \left.-\psi_{Y, T i, j+1}^{k+1}+\psi_{Y, T i+1, j+1}^{k}+\psi_{Y, T i+1, j}^{k}-\psi_{Y, T i, j+1}^{k}-\psi_{Y, T i, j}^{k}\right]-0.30313\left\{\frac { 1 } { 4 r } \left[M_{X i+1, j+1}^{k+1}\right.\right. \\
& \left.\left.+M_{X i+1, j}^{k+1}+M_{X i, j+1}^{k+1}+M_{X i, j}^{k+1}-M_{X i+1, j+1}^{k}-M_{X i+1, j}^{k}-M_{X i, j+1}^{k}-M_{X i, j}^{k}\right]\right\} \\
& -1.66722\left\{\frac { 1 } { 4 k } \left[M_{X i+1, j+1}^{k+1}+M_{X i+1, j}^{k+1}-M_{X i, j}^{k+1}-M_{X i, j+1}^{k+1}+M_{X i+1, j+1}^{k}+M_{X i+1, j}^{k}\right.\right. \\
& \left.\left.-M_{X i, j+1}^{k}-M_{X i, j}^{k}\right]\right\}+0.30313\left\{\frac { 1 } { 4 r } \left[M_{X i+1, j+1}^{k+1}+M_{X i+1, j}^{k+1}+M_{X i, j+1}^{k+1}+M_{X i, j}^{k+1}\right.\right. \\
& \left.\left.-M_{X i+1, j+1}^{k}-M_{X i+1, j}^{k}-M_{X i, j+1}^{k}-M_{X i, j}^{k}\right]\right\}+1.66722\left\{\frac { 1 } { 4 h } \left[M_{X i+1, j+1}^{k+1}\right.\right. \\
& \left.\left.+M_{X i+1, j}^{k+1}-M_{X i, j}^{k+1}-M_{X i, j+1}^{k+1}+M_{X i+1, j+1}^{k}+M_{X i+1, j}^{k}-M_{X i, j+1}^{k}-M_{X i, j}^{k}\right]\right\} \\
& 0.25\left[Q_{x i+1, j+1}^{k+1}+Q_{x i+1, j}^{k+1}-Q_{x i, j}^{k+1}-Q_{x i, j+1}^{k+1}+Q_{x i+1, j+1}^{k}+Q_{x i+1, j}^{k}-Q_{x i, j+1}^{k}-Q_{x i, j}^{k}\right] \\
& +0.25\left[Q_{x i+1, j+1}^{k+1}+Q_{x i, j+1}^{k+1}-Q_{x i+1, j}^{k+1}-Q_{x i, j}^{k+1}+Q_{x i+1, j+1}^{k}+Q_{x i, j+1}^{k}-Q_{x i+1, j}^{k}-Q_{x i, j}^{k}\right] \\
& +12.5\left[W_{x i+1, j+1}^{k+1}+W_{x i, j+1}^{k+1}+W_{x i+1, j}^{k+1}+W_{x i, j}^{k+1}+W_{x i+1, j+1}^{k}+W_{x i, j+1}^{k}+W_{x i+1, j}^{k}+W_{x i, j}^{k}\right] \\
& +1.25\left[D_{T i+1, j+1}^{k+1}+D_{T i+1, j}^{k+1}+D_{T i, j}^{k+1}+D_{T i, j+1}^{k+1}-D_{T i+1, j+1}^{k}-D_{T i+1, j}^{k}-D_{T i, j+1}^{k}-D_{T i, j}^{k}\right] \\
& +2.5\left[D_{x i+1, j+1}^{k+1}+D_{x i+1, j}^{k+1}-D_{x i, j}^{k+1}-D_{x i, j+1}^{k+1}+D_{x i+1, j+1}^{k}+D_{x i+1, j}^{k}-D_{x i, j+1}^{k}-D_{x i, j}^{k}\right]
\end{aligned}
$$




$$
\begin{aligned}
& +2.5\left[D_{Y i+1, j+1}^{k+1}+D_{Y i, j+1}^{k+1}-D_{Y i+1, j}^{k+1}-D_{Y i, j}^{k+1}+D_{Y i+1, j+1}^{k}+D_{Y i, j+1}^{k}-D_{Y i+1, j}^{k}-D_{Y i, j}^{k}\right] \\
& +0.008335\left[g \sin \theta+\frac{1}{4}\left[D_{T i+1, j+1}^{k+1}+D_{T i+1, j}^{k+1}+D_{T i, j}^{k+1}+D_{T i, j+1}^{k+1}-D_{T i+1, j+1}^{k}-D_{T i+1, j}^{k}\right.\right. \\
& \left.\left.-D_{T i, j+1}^{k}-D_{T i, j}^{k}\right]\right]+0.011459\left[D_{T i+1, j+1}^{k+1}+D_{T i+1, j}^{k+1}+D_{T i, j}^{k+1}+D_{T i, j+1}^{k+1}-D_{T i+1, j+1}^{k}\right. \\
& \left.-D_{T i+1, j}^{k}-D_{T i, j+1}^{k}-D_{T i, j}^{k}\right]+0.005729\left[\psi_{X, T i+1, j+1}^{k+1}+\psi_{X, T i, j+1}^{k+1}+\psi_{X, T i+1, j}^{k+1}\right. \\
& \left.+\psi_{X, T i, j}^{k+1}+\psi_{X, T i+1, j+1}^{k}+\psi_{X, T i, j+1}^{k}+\psi_{X, T i+1, j}^{k}+\psi_{X, T i, j}^{k}\right]-0.052101\left[M_{x i+1, j+1}^{k+1}\right. \\
& \left.+M_{x i, j+1}^{k+1}+M_{x i+1, j}^{k+1}+M_{x i, j}^{k+1}+M_{x i+1, j+1}^{k}+M_{x i, j+1}^{k}+M_{x i+1, j}^{k}+M_{x i, j}^{k}\right] \\
& +0.01042\left[M_{Y i+1, j+1}^{k+1}+M_{Y i, j+1}^{k+1}+M_{Y i+1, j}^{k+1}+M_{Y i, j}^{k+1}+M_{Y i+1, j+1}^{k}+M_{Y i, j+1}^{k}\right. \\
& \left.+M_{Y i+1, j}^{k}+M_{Y i, j}^{k}\right]-0.005729\left[Q_{x i+1, j+1}^{k+1}+Q_{x i+1, j}^{k+1}+Q_{x i, j+1}^{k+1}+Q_{x i, j}^{k+1}-Q_{x i+1, j+1}^{k}\right. \\
& \left.-Q_{x i+1, j}^{k}-Q_{x i, j+1}^{k}-Q_{x i, j}^{k}\right]-0.0315105\left[Q_{Y i+1, j+1}^{k+1}+Q_{Y i+1, j}^{k+1}-Q_{Y i, j+1}^{k+1}-Q_{Y i, j}^{k+1}\right. \\
& \left.+Q_{Y i+1, j+1}^{k}+Q_{Y i+1, j}^{k}-Q_{Y i, j+1}^{k}-Q_{Y i, j}^{k}\right]=0 \\
& 0.25\left[M_{x i+1, j+1}^{k+1}+M_{x i+1, j}^{k+1}+M_{x i, j+1}^{k+1}+M_{x i, j}^{k+1}-M_{x i+1, j+1}^{k}-M_{x i+1, j}^{k}-M_{x i, j+1}^{k}-M_{x i, j}^{k}\right] \\
& =-0.63\left[\frac { 1 } { 4 } \left[\begin{array}{c}
k+1 \\
X, T i+1, j+1
\end{array}+\psi_{X, T i+1, j}^{k+1}-\psi_{X, T i, j}^{k+1}-\psi_{X, T i, j+1}^{k+1}+\psi_{X, T i+1, j+1}^{k}\right.\right. \\
& \left.\left.+\psi_{X, T i+1, j}^{k}-\psi_{X, T i, j+1}^{k}-\psi_{X, T i, j}^{k}\right]\right]-0.126\left[\frac { 1 } { 4 } \left[\psi_{X, T i+1, j+1}^{k+1}+\psi_{X, T i, j+1}^{k+1}\right.\right. \\
& \left.\left.-\psi_{X, T i+1, j}^{k+1}-\psi_{X, T i, j}^{k+1}+\psi_{X, T i+1, j+1}^{k}+\psi_{X, T i, j+1}^{k}-\psi_{X, T i+1, j}^{k}-\psi_{X, T i, j}^{k}\right]\right] \\
& 0.25\left[M_{Y i+1, j+1}^{k+1}+M_{Y i+1, j}^{k+1}+M_{Y i, j+1}^{k+1}+M_{Y i, j}^{k+1}-M_{Y i+1, j+1}^{k}-M_{Y i+1, j}^{k}-M_{Y i, j+1}^{k}-M_{Y i, j}^{k}\right] \\
& =-0.63\left[\frac { 1 } { 4 } \left[\psi_{X, T i+1, j+1}^{k+1}+\psi_{X, T i, j+1}^{k+1}-\psi_{X, T i+1, j}^{k+1}-\psi_{X, T i, j}^{k+1}+\psi_{X, T i+1, j+1}^{k}\right.\right. \\
& \left.\left.+\psi_{X, T i, j+1}^{k}-\psi_{X, T i+1, j}^{k}-\psi_{X, T i, j}^{k}\right]\right]-0.126\left[\frac { 1 } { 4 } \left[\begin{array}{c}
k+1 \\
\psi_{X, T i+1, j+1}^{k}
\end{array}+\psi_{X, T i, j+1}^{k+1}\right.\right. \\
& \left.\left.-\psi_{X, T i+1, j}^{k+1}-\psi_{X, T i, j}^{k+1}+\underset{X, T i+1, j+1}{k}+\psi_{X, T i, j+1}^{k}-\psi_{X, T i+1, j}^{k}-\psi_{X, T i, j}^{k}\right]\right] \\
& 0.25\left[M_{X Y i+1, j+1}^{k+1}+M_{Y i+1, j}^{k+1}+M_{Y i, j+1}^{k+1}+M_{Y i, j}^{k+1}-M_{Y i+1, j+1}^{k}-M_{Y i+1, j}^{k}-M_{Y i, j+1}^{k}-M_{Y i, j}^{k}\right] \\
& =-0.252\left[\frac { 1 } { 4 } \left[\begin{array}{c}
k+1 \\
\psi_{X, T i+1, j+1}
\end{array}+\psi_{X, T i, j+1}^{k+1}-\psi_{X, T i+1, j}^{k+1}-\psi_{X, T i, j}^{k+1}+\psi_{X, T i+1, j+1}^{k}\right.\right. \\
& \left.\left.+\psi_{X, T i, j+1}^{k}-\psi_{X, T i+1, j}^{k}-\psi_{X, T i, j}^{k}\right]\right]+0.252\left[\frac { 1 } { 4 } \left[\begin{array}{c}
k+1 \\
\psi_{Y, T i+1, j+1}^{k}
\end{array}+\psi_{Y, T i, j+1}^{k+1}\right.\right. \\
& \left.\left.-\psi_{Y, T i+1, j}^{k+1}-\psi_{Y, T i, j}^{k+1}+\underset{Y, T i+1, j+1}{k}+\psi_{Y, T i, j+1}^{k}-\psi_{Y, T i+1, j}^{k}-\psi_{Y, T i, j}^{k}\right]\right] \\
& 0.25\left[Q_{x i+1, j+1}^{k+1}+Q_{x i+1, j}^{k+1}+Q_{x i, j}^{k+1}+Q_{x i, j+1}^{k+1}-Q_{x i+1, j+1}^{k}-Q_{x i+1, j}^{k}-Q_{x i, j+1}^{k}-Q_{x i, j}^{k}\right] \\
& =2\left[\frac { 1 } { 8 } \left[\psi_{X, T i+1, j+1}^{k+1}+\psi_{X, T i+1, j}^{k+1}+\psi_{X, T i, j}^{k+1}+\psi_{X, T i, j+1}^{k+1}+\psi_{X, T i+1, j+1}^{k}\right.\right. \\
& \left.\left.+\psi_{X, T i+1, j}^{k}+\psi_{X, T i, j+1}^{k}+\psi_{X, T i, j}^{k}\right]\right]-2\left[\frac { 1 } { 4 } \left[D_{Y i+1, j+1}^{k+1}+D_{Y i+1, j}^{k+1}\right.\right. \\
& \left.\left.-D_{Y i, j}^{k+1}-D_{Y i, j+1}^{k+1}+D_{Y i+1, j+1}^{k}+D_{Y i+1, j}^{k}-D_{Y i, j+1}^{k}-D_{Y i, j}^{k}\right]\right]
\end{aligned}
$$




$$
\begin{aligned}
& 0 . 25\left[Q_{Y i+1, j+1}^{k+1}+Q_{Y i+1, j}^{k+1}+Q_{Y i, j}^{k+1}+Q_{Y i, j+1}^{k+1}-Q_{Y i+1, j+1}^{k}-Q_{Y i+1, j}^{k}-Q_{Y i, j+1}^{k}-Q_{Y i, j}^{k}\right] \\
&= 2\left[\frac { 1 } { 8 } \left[\psi_{Y, T i+1, j+1}^{k+1}+\psi_{Y, T i+1, j}^{k+1}+\psi_{Y, T i, j}^{k+1}+\psi_{Y, T i, j+1}^{k+1}+\psi_{Y, T i+1, j+1}^{k}\right.\right. \\
&\left.\left.+\psi_{Y, T i+1, j}^{k}+\psi_{Y, T i, j+1}^{k}+\psi_{Y, T i, j}^{k}\right]\right]-2\left[\frac { 1 } { 4 } \left[D_{Y i+1, j+1}^{k+1}+D_{Y i, j+1}^{k+1}\right.\right. \\
&\left.\left.-D_{Y i+1, j}^{k+1}-D_{Y i, j}^{k+1}+D_{Y i+1, j+1}^{k}+D_{Y i, j+1}^{k}-D_{Y i+1, j}^{k}-D_{Y i, j}^{k}\right]\right] \\
& 0.125\left[D_{T i+1, j+1}^{k+1}+D_{T i+1, j}^{k+1}+D_{T i, j}^{k+1}+D_{T i, j+1}^{k+1}-D_{T i+1, j+1}^{k}-D_{T i+1, j}^{k}-D_{T i, j+1}^{k}-D_{T i, j}^{k}\right] \\
&=0.25\left[W_{x i+1, j+1}^{k+1}+W_{x i, j+1}^{k+1}+W_{x i+1, j}^{k+1}+W_{x i, j}^{k+1}-W_{x i+1, j+1}^{k}-W_{x i, j+1}^{k}-W_{x i+1, j}^{k}-W_{x i, j}^{k}\right] \\
& 0.125\left[D_{Y i+1, j+1}^{k+1}+D_{Y i+1, j}^{k+1}+D_{Y i, j}^{k+1}+D_{Y i, j+1}^{k+1}+D_{Y i+1, j+1}^{k}+D_{Y i+1, j}^{k}+D_{Y i, j+1}^{k}+D_{Y i, j}^{k}\right] \\
&=0.25\left[W_{x i+1, j+1}^{k+1}+W_{x i+1, j}^{k+1}-W_{x i, j}^{k+1}-W_{x i, j+1}^{k+1}+W_{x i+1, j+1}^{k}+W_{x i+1, j}^{k}-W_{x i, j+1}^{k}-W_{x i, j}^{k}\right] \\
& 0.125\left[D_{T i+1, j+1}^{k+1}+D_{T i+1, j}^{k+1}+D_{T i, j}^{k+1}+D_{T i, j+1}^{k+1}+D_{T i+1, j+1}^{k}+D_{T i+1, j}^{k}+D_{T i, j+1}^{k}+D_{T i, j}^{k}\right] \\
&=0.25\left[W_{x i+1, j+1}^{k+1}+W_{x i, j+1}^{k+1}-W_{x i+1, j}^{k+1}-W_{x i, j}^{k+1}+W_{x i+1, j+1}^{k}+W_{x i, j+1}^{k}-W_{x i+1, j}^{k}-W_{x i, j}^{k}\right]
\end{aligned}
$$

The matrices now appear as follows:

$$
R_{i, j+1} S_{i, j+1}^{\prime}=\left[\begin{array}{ccccccccccc}
0.3 & 0 & 0.3 & 0.1 & 0 & 0 & 0 & 0 & 0 & 0 & 0 \\
0.3 & 0 & -0.3 & 0 & 0.1 & 0 & 0.1 & 0 & 0 & 0 & 0 \\
-0.1 & 0 & 0 & 0 & 0 & 0 & 0 & 0 & -1.3 & -2.5 & 2.5 \\
-1.3 & 0 & 0 & 0 & 0 & -0.1 & 0 & 0 & 0 & 0 & 0 \\
0 & -0.3 & 0 & 0 & 0 & 0.2 & 0 & 0 & 0 & 0 & 0 \\
0 & 0 & -0.3 & 0.1 & -0.1 & 0 & 0 & 0 & 0 & 0 & 0 \\
0 & 0 & 0 & -0.3 & 0 & -0.3 & 0 & -0.5 & 0 & 0 & 0 \\
0 & 0 & 0 & 0 & -0.3 & 0 & -0.3 & 0.5 & 0 & 0 & 0 \\
0 & 0 & 0 & 0 & 0 & 0 & 0 & -0.1 & 0.3 & 0 & 0 \\
0 & 0 & 0 & 0 & 0 & 0 & 0 & 0.1 & -0.3 & 0 & 0 \\
0 & 0 & 0 & 0 & 0 & 0 & 0 & 0.1 & -0.3 & 0 & 0
\end{array}\right]\left[\begin{array}{c}
M_{X_{i, j+1}} \\
M_{Y_{i, j+1}} \\
M_{X Y_{i, j+1}} \\
Q_{X_{i, j+1}} \\
Q_{Y_{i, j+1}} \\
\psi_{X T_{i, j+1}} \\
\psi_{Y T_{i, j+1}} \\
D_{T_{i, j+1}} \\
W_{i, j+1} \\
D_{X_{i, j+1}} \\
D_{Y_{i, j+1}}
\end{array}\right]
$$

The above is at a particular node $(i, j+1)$. Similar matrices can be shown for the other nodes, but for brevity sake.

\section{Results Discussion}

The paper set out to analyse, numerically, the vibration of rectangular elastic orthotropic damped inclined Mindlin plate, because of applied force, using finite difference method. The plate was supported by a Pasternak foundation. Deflection of the plate was calculated for specific values of foundation parameter and contact area of the plate. It was observed that Mindlin plate has highest maximum amplitude when compared with Non-Mindlin plate. The response maximum amplitude decreases with an increase in the value of the subgrade's shear modulus for fixed value of foundation stiffness, contact area and velocity. It was noticed that the response amplitude of the plate continuously supported 
by a Pasternak foundation is less than that of the plate not resting on any elastic subgrade. As the foundation stiffness and shear modulus increase the response amplitude decreases. Also, it was observed that as the contact area increases the response maximum amplitude increases with fixed values of the foundation stiffness and the subgrade's shear modulus. Finally it was observed that the maximum amplitude increases as the velocity increases.

\section{Conclusion}

The structure of interest was an inclined Mindlin rectangular plate on Pasternak elastic foundation, under the influence of a uniform partially distributed moving load. The problem was to use finite difference technique to solve the governing equation of a moving load problem. The dynamic response of the whole system was determined by solving the resulting first order coupled partial differential equations obtained from governing equations for the simply supported Mindlin plate. The study has contributed to scientific knowledge by showing that Pasternak foundation, on which the inclined Mindlin plate rests, has a significance effect on the dynamic response of the plate to a partially distributed moving load. The effect of rotating inertia and shear deformation on the dynamic response of the inclined Mindlin plate to the moving load gives more realistic results for practical application, especially when such inclined plate is supported by a Pasternak type of subgrade foundation.

\section{References}

[1] Rossi Thomas, D. (1982) Chladin's Law for Vibrating Plates. American Journal of Physics, 50, 3.

[2] Ladislaw, F. (1972) Vibration of Solids and Structures under Moving Loads. Noordhoff International Publishing, Groningen.

[3] Civalek, O. (2005) Large Deflection Static and Dynamic Analysis of Thin Circular Plates Resting on Two Parameter Elastic Foundation HDQ/FO Couple Methodology Approaches. International Journal of Computational Mechanics, 2, 271-291.

[4] Civalek, O. and Yavas, A. (2006) Large Deflection Static Analysis of Rectangular Plate on Two Parameter Elastic Foundation. International Journal of Science and Technology, 1, 43-50.

[5] Dowell, E.H. (1974) Dynamic Analysis of an Elastic Plate on a Thin Elastic Foundation. Journal of Sound and Vibration, 35, 343-360. https://doi.org/10.1016/0022-460X(74)90065-0

[6] Gbadeyan, J.A. and Dada, M.S. (2006) Dynamic Response of a Mindlin Elastic Rectangular Plate under a Distributed Moving Mass. International Journal of Mechanical Science, 48, 323-340. https://doi.org/10.1016/j.ijmecsci.2005.09.005

[7] Gbedeyan, J.A. and Dada, M.S. (2001) The Dynamic Response of Plates on Pasternak Foundation to Distributed Moving Load. Journal of the Nigerian Association of Mathematical Physics, 5, 185-200.

[8] Agarana, M.C. and Gbadeyan, J.A. (2016) Application of Differential Transform Method to Vibration Analysis of damped railway bridge on Pastwernak Foundation under Moving Train. 2016 Proceedings of the World Congress on Engineering, 
London, 29 June-1 July 2016, 1177-1179.

[9] Gerg, V.K. and Dukkipati, R.V. (1984) Dynamics of Railway Vehicle Systems. Academic Press, New York.

[10] Agarana, M.C., Gbadeyan, J.A., Agboola, O.O., Anake, T. and Adeleke, O.J. (2015) Dynamic Response of an Inclined Railway Bridge Supported by Winkler Foundation under a Moving Railway Vehicle. Australian Journal of Basic and Applied Sciences, 9, 335-361.

[11] Mindlin, R.D. (1957) Influence of Rotatory Inertia and Shear on Flexural Motions of Isotropic Elastic Plates. Journal of Applied Mechanics, 18, 31-38.

[12] Boay, C.G. (1993) Free Vibration of Rectangular Isotropic Plates with or without a Concentrated Mass. Computers and Structures, 48, 529-533. https://doi.org/10.1016/0045-7949(93)90331-7

[13] Gbadeyan, J.A. and Agarana, M.C. (2014) Dynamic Analysis of Railway Bridges Supported by Winkler Foundation under Uniform Partially Distributed Moving Railway Vehicle. International Journal of Sustainable Development and Planning, 135, 873-883. https://doi.org/10.2495/CR140731

[14] Agarana, M.C., Gbadeyan, J.A. and Ajayi, O.O. (2016) Dynamic Response of Inclined Isotropic Elastic Damped Rectangular Mindlin Plate resting on Pasternak Foundation under a Moving Load. 2016 Proceedings of the International MultiConference of Engineers and Computer Scientists, Hong Kong, 16-18 March 2016, 713-718.

[15] Agarana, M.C., Gbadeyan, J.A. and Ajayi, O.O. (2016) Dynamic Response of Mindlin Elastic Plate Supported by Pasternak Foundation under Uniform Partially Distributed Moving Load. Australian Journal of Applied, 2, 17-26.

[16] Nguyan-Thoi, T., Luong-Van, H., Phung-Van, P., Rabczuk, T. and Tran-Trung, D. (2013) Dynamic Responses of Composite Plates on the Paternak Foundation Subjected to a Moving Mass by a Cell-Based Smoothed Discrete Shear Gap (CS-FEM-DSG3) Method. International Journal of Composite Materials, 3, 19-27.

[17] Agarana, M.C. and Ede, A. (2016) Free Vibration Analysis of Elastic Orthotropic Rectangular Inclined Damped Highway Supported by Pasternak Foundation under Moving Aerodynamic Automobile. 2016 Proceedings of the World Congress on Engineering, London, 29 June-1 July 2016, 978-981.

[18] Agarana, M.C. and Gbadeyan, J.A. (2015) On Response of Elastic Isotropic Damped Shear Highway Bridge Supported by Sub-Grade to Uniform Partially Distributed Moving Vehicle. International Journal of Applied Engineering Research, 10, 3979-3981.

[19] Amiri, J.V., Nikkho, A., Dnvoodi, M.R. and Hassanabadi, M.E. (2013) Vibration Analysis of a Mindlin Elastic Plate under a Moving Mass Excitation by Eigenfuntion Expansion Method. Thin- Walled Structure, 62, 53-64. https://doi.org/10.1016/j.tws.2012.07.014

[20] Arnoid, D.K. (1964) Elastic and Viscoelastic Foundation Models. Journal of Applied Mechanics Transactions of the ASME, 33, 491-498.

[21] Agarana, M.C. and Gbadeyan, J.A. (2015) Finite Difference Dynamic Analysis of Railway Bridges Supported by Pasternak Foundation under Uniform Partially Distributed Moving Railway Vehicle. Proceedings of the World Congress on Engineering and Computer Science, San Francisco, 21-23 October 2015, 996-1000.

[22] Emetere, M.E., Samuel, S.E. and Michael, A.C. (2016) Virtual Observation of Fermtosecond Spin Dynamics Mechanism in Grapheme. 2016 Proceedings of the World Congress on Engineering, London, 29 June-1 July 2016, 658-660. 